TAIWANESE JOURNAL OF MATHEMATICS

Vol. 10, No. 4, pp. 1015-1023, June 2006

This paper is available online at http://www.math.nthu.edu.tw/tjm/

\title{
COLORING THE SQUARE OF AN OUTERPLANAR GRAPH
}

\author{
Ko-Wei Lih and Wei-Fan Wang
}

\begin{abstract}
Let $G$ be an outerplanar graph with maximum degree $\Delta(G) \geq 3$. We prove that the chromatic number $\chi\left(G^{2}\right)$ of the square of $G$ is at most $\Delta(G)+2$. This confirms a conjecture of Wegner [8] for outerplanar graphs. The upper bound can be further reduced to the optimal value $\Delta(G)+1$ when $\Delta(G) \geq 7$.
\end{abstract}

\section{INTRODUCTION}

Only simple graphs are considered in this paper. For two vertices $u$ and $v$ of a graph $G(V, E)$, let $\operatorname{dist}_{G}(u, v)$ denote the distance between $u$ and $v$ in $G$, that is the length of a shortest path connecting them. The square $G^{2}$ of a graph $G$ is the graph defined on the vertex set $V(G)$ such that $u$ and $v$ are adjacent in $G^{2}$ if and only if $1 \leq \operatorname{dist}_{G}(u, v) \leq 2$. A proper $k$-coloring is a mapping $\phi$ from $V(G)$ to the set $\{1,2, \ldots, k\}$ such that $\phi(u) \neq \phi(v)$ whenever $u$ and $v$ are adjacent. Obviously, a $k$-coloring $\phi$ of $G$ gives rise to a proper coloring of $G^{2}$ if and only if $\phi(u) \neq \phi(v)$ whenever $1 \leq \operatorname{dist}_{G}(u, v) \leq 2$. We call such a coloring $\phi$ a square-k-coloring of $G$. The chromatic number $\chi(G)$ is the least number $k$ such that $G$ admits a proper $k$-coloring. Let $\Delta(G)$ denote the maximum degree of a vertex of the graph $G$. It is evident that $\chi\left(G^{2}\right) \geq \Delta(G)+1$ for any graph $G$. This lower bound is sharp. For instance, $\chi\left(T^{2}\right)=\Delta(T)+1$ for every tree $T$ with at least one edge. On the other hand, it is easy to see that $\chi\left(G^{2}\right) \leq \Delta^{2}(G)+1$ for any graph $G$. This upper bound is also sharp. The 5-cycle and the Petersen graph are two examples.

Wegner [8] first investigated the chromatic number of the square of a planar graph. He proved that $\chi\left(G^{2}\right) \leq 8$ for every planar graph $G$ with $\Delta(G)=3$ and conjectured that the upper bound could be reduced to 7. Recently, Thomassen [6] has established Wegner's conjecture. Wegner [8] also proposed the following conjecture. The upper bounds are sharp if the conjecture is true.

Received July 16, 2004, accepted July 27, 2004.

Communicated by Xuding Zhu.

2000 Mathematics Subject Classification: 05C15.

Key words and phrases: Outerplanar graph, Square, Chromatic number. 
Conjecture 1. Let $G$ be a planar graph. Then

$$
\chi\left(G^{2}\right) \leq \begin{cases}\Delta(G)+5 & \text { if } 4 \leq \Delta(G) \leq 7 \\ \lfloor 3 \Delta(G) / 2\rfloor+1 & \text { if } \Delta(G) \geq 8 .\end{cases}
$$

This conjecture remains open. The best upper bound as far as we know is $5 \Delta(G) / 3+78$ established by Molloy and Salavatipour [5]. This improves other recently obtained upper bounds: $\lfloor 9 \Delta / 5\rfloor+2$ for $\Delta(G) \geq 749$ ([1]), $\lceil 9 \Delta / 5\rceil+1$ for $\Delta(G) \geq 47$ ([2]), and $2 \Delta(G)+25$ ([4]). For planar graphs of large girth, better upper bounds for $\chi\left(G^{2}\right)$ are known. Wang and Lih [7] proved that if $G$ is a planar graph with girth $g(G)$, then $\chi\left(G^{2}\right) \leq \Delta(G)+5$ when $g(G) \geq 7$, $\chi\left(G^{2}\right) \leq \Delta(G)+10$ when $g(G) \geq 6$, and $\chi\left(G^{2}\right) \leq \Delta(G)+16$ when $g(G) \geq 5$.

The focus of this paper is to study the chromatic number of the square of an outerplanar graph. A planar graph is said to be outerplanar if it has a plane embedding such that all vertices lie on the boundary of the unbounded face. An outerplane graph is a particular embedding of an outerplanar graph. Bodlaender et al. [3] showed that there are polynomial time algorithms for coloring the square of an outerplanar graph $G$ and $\chi\left(G^{2}\right) \leq \Delta(G)+5$. We will reduce the upper bound to $\Delta(G)+2$ when $\Delta(G) \geq 3$, and even to the optimal result $\chi\left(G^{2}\right)=\Delta(G)+1$ when $\Delta(G) \geq 7$.

\section{Special Vertices of Degree 2}

A vertex of degree $k$ is called a $k$-vertex. The degree of $v$ in the graph $G$ is denoted by $d_{G}(v)$. For a vertex $v$ of a graph $G$, define $N_{i}^{G}(v)=\{u \in V(G) \mid$ $\left.\operatorname{dist}_{G}(u, v)=i\right\}$ for $i \geq 1$ and define $\beta_{G}(v)=\left|N_{1}^{G}(v)\right|+\left|N_{2}^{G}(v)\right|$.

For an outerplane graph $G$, all faces are called inner faces, except that the unbounded one is called the outer face. The boundary edges of the outer face are called outer edges. All other edges are called inner edges. If $G$ is 2-connected and $\Delta(G) \geq 3$, then an inner face $f$ of $G$ is called an end face if the boundary of $f$ contains exactly one inner edge, i.e., the boundary of $f$ contains exactly two vertices of degree 3 or more. The dual graph of $G$ becomes a tree of order at least 2 when the vertex corresponding to the outer face is deleted. Thus there exist at least two leaves that determine two end faces of $G$.

Let $|G|$ denote the order of $G$. It is well-known that a 2-connected outerplane graph $G$ has at least one 2-vertex if $|G| \geq 3$, and at least two nonadjacent 2-vertices if $|G| \geq 4$.

Let $M_{3}$ be the graph obtained from a path $x_{1}, x_{2}, \ldots, x_{7}$ of length 6 , where $x_{1} \neq x_{7}$, by adding the edges $x_{1} x_{3}, x_{3} x_{5}$, and $x_{5} x_{7}$. A graph $G$ is said to contain the configuration $M_{3}$ if $M_{3}$ appears in $G$ as a subgraph such that $d_{G}\left(x_{i}\right)=2$ 
for $i=2,4,6$ and $d_{G}\left(x_{j}\right)=4$ for $j=1,3,5,7$. Define the function $f$ so that $f(\Delta)=\Delta+1$ when $3 \leq \Delta \leq 6$, and $f(\Delta)=\Delta$ when $\Delta \geq 7$.

Theorem 1. Let $G$ be a 2-connected outerplane graph with $|G| \geq 3$. Suppose that $\Delta(G) \geq 3$ and $G$ does not contain the configuration $M_{3}$ when $\Delta(G)=4$. Then there exists a vertex $u$ of degree 2 such that $\beta_{G}(u) \leq f(\Delta)$.

Proof. Let $\Delta=\Delta(G)$. If $\Delta=3$, then it is easy to see that $\beta_{G}(u) \leq 4$ for some 2-vertex in the boundary of an end face.

So we may assume that $\Delta \geq 4$. The smallest 2-connected outerplane graph with maximum degree $\Delta$ consists of a vertex joined to every vertex of a path of length $\Delta-1$. Evidently, this graph has a 2-vertex $u$ such that $\beta_{G}(u)=\Delta \leq f(\Delta)$.

We now proceed by induction on $|G|$. Let $C$ be the cycle consisting of all the outer edges.

Since $\Delta \geq 4$, there exists a subpath $P$ of $C$ of length at least 2 whose ends have degree at least 3 in $G$, but all of whose internal vertices have degree 2 in $G$. If the length of $P$ is at least 3 , then we may contract the second edge from one end of $P$ to get a shorter path. The result then follows by induction. Therefore we assume that no two 2-vertices in $C$ are adjacent.

Let an arbitrary 2-vertex $u$ of $G$ have neighbors $v$ and $w$. If $v$ and $w$ are not adjacent and their degrees are both less than $\Delta$, then we add a new edge $v w$. In so doing, we do not change the value of $\beta_{G}(u)$. We perform such additions wherever possible. So we may assume the following for $G$.

Convention. Let $u$ be a 2-vertex with neighbors $v$ and $w$ such that $d_{G}(v) \geq$ $d_{G}(w)$. If $v$ and $w$ are not adjacent, then $d_{G}(v)=\Delta$.

In the sequel, we always label the two neighbors $v$ and $w$ of a 2-vertex $u$ in such a way that $d_{G}(v) \geq d_{G}(w)$. Let $x_{v}$ and $x_{w}$ denote the neighbors in $C-u$ of $v$ and $w$, respectively. Let $P(u): z_{0}, z_{1}, \ldots, z_{t}$ be the shortest subpath of $C$ containing the path $x_{v}, v, u, w, x_{w}$ and containing all the vertices in $N_{1}^{G}(v) \cup N_{1}^{G}(w)$. Then there exists an index $i$ such that $z_{i-2}=x_{v}, z_{i-1}=v, z_{i}=u, z_{i+1}=w$, and $z_{i+2}=x_{w}$. Note that $z_{0}$ and $z_{t}$ are in $N_{1}^{G}(v) \cup N_{1}^{G}(w)$. Now we choose a vertex $u$ such that $P(u)$ has the minimum length.

Case 1. The neighbors of $u$ are not adjacent.

Then by the Convention, $d_{G}(v)=\Delta$. Let $H$ be the graph obtained from $G$ by deleting $u$ and adding the edge $v w$. We see that $|H|<|G|, \Delta(H)=\Delta(G)$, and $H$ satisfies the assumptions of the theorem. By the induction hypothesis, there exists a vertex $x$ such that $d_{H}(x)=2$ and $\beta_{H}(x) \leq f(\Delta)$. Since $d_{H}(x)=2$ and both $d_{H}(v)$ and $d_{H}(w)$ are at least $3, x$ is different from $v$ and $w$. Obviously, at 
most one of $v$ and $w$ may be a neighbor of $x$. Suppose that both $v$ and $w$ are not neighbors of $x$. Then $\beta_{G}(x)=\beta_{H}(x)$ and $x$ is what we are looking for.

Suppose that $x$ is adjacent to $v$ and $z$ for some $z$ different from $w$. If $z$ is not a neighbor of $w$, then $N_{2}^{G}(x)=\left(N_{2}^{H}(x) \backslash\{w\}\right) \cup\{u\}$. Again, $\beta_{G}(x)=\beta_{H}(x)$. If $z$ is a neighbor of $w$, then it implies that $d_{G}(v)=\Delta \leq 3$, contradicting our present assumption that $\Delta \geq 4$.

Suppose that $x$ is adjacent to $w$ and $z$ for some $z$ different from $v$. If $z$ is not a neighbor of $v$, then $N_{2}^{G}(x)=\left(N_{2}^{H}(x) \backslash\{v\}\right) \cup\{u\}$. Again $\beta_{G}(x)=\beta_{H}(x)$. If $z$ is a neighbor of $v$, then $z$ and $w$ must be adjacent. Now let the neighbor of $z$ in $C-x$ be $y$, where $y \neq v$ as $d_{G}(v)=\Delta \geq 4$. Hence $d_{G}(z) \geq 4$. If $y$ is adjacent to $v$, then $\beta_{G}(x)=5 \leq f(\Delta)$, and we are done. Suppose that $y$ is not a neighbor of $v$. If $d_{G}(v)=4$, then $d_{G}(z)=4$ since $d_{G}(v)=\Delta$. Again, it follows that $\beta_{G}(x)=5 \leq f(\Delta)$, and we are done.

Suppose next $d_{G}(v) \geq 5$. Let $j \leq i-2$ be the largest index such that $z_{j}$ is a neighbor of $v$. If $j=0$, then all the vertices at distance at most 2 from $x$ are included in the path $z_{i-1}, z_{i}, \ldots, z_{t}$. It follows that $P(x)$ is strictly shorter than $P(u)$, a contradiction. Hence $j>0$. Let $k$ be the smallest index, $j<k \leq i-2$, such that $z_{k}$ is a 2-vertex. If $k<i-2$, then all the vertices at distance at most 2 from $z_{k}$ are included in the path $z_{0}, z_{1}, \ldots, z_{i-1}$. It follows that $P\left(z_{k}\right)$ is strictly shorter than $P(u)$, a contradiction. Now suppose that $k=i-2$. In this case, $z_{i-2}$ and $z_{j}$ must be adjacent, i.e., $j=i-3$. If $z_{j-1}$ is also a neighbor of $v$, then $\beta_{G}\left(z_{i-2}\right)=d_{G}(v) \leq f(\Delta)$.

Suppose that $z_{j-1}$ is not a neighbor of $v$. If there is at least one vertex $z_{p}$, $0<p<j-1$, that is adjacent to $v$, then there is some 2-vertex $z_{m}, p<m<j$, such that all the vertices at distance at most 2 from $z_{m}$ are included in the path $z_{0}$, $z_{1}, \ldots, z_{i-1}$. Therefore, $P\left(z_{m}\right)$ is strictly shorter than $P(u)$, a contradiction. Now suppose that no such $z_{p}$ exists. It implies that $d_{G}(v)=\Delta=5$. Hence, $d_{G}(z) \leq 5$. It follows that $\beta_{G}(x) \leq 6=f(\Delta)$, and we are done.

Case 2. The neighbors of $u$ are adjacent.

If $v$ is adjacent to $x_{w}$, then $\beta_{G}(u)=d_{G}(v) \leq \Delta \leq f(\Delta)$ and $u$ is what we are looking for. Henceforth we assume that $v$ and $x_{w}$ are not adjacent.

Subcase 2.1. The number of indices $j, 0 \leq j<i-2$, such that $z_{j}$ is adjacent to $v$ is at least two.

Let $j<i-2$ be the largest index such that $z_{j}$ is a neighbor of $v$. Let $k$ be the smallest index, $j<k \leq i-2$, such that $z_{k}$ is a 2 -vertex. If $k<i-2$ or $z_{t}$ is not adjacent to $v$, then all the vertices at distance at most 2 from $z_{k}$ are included in the path $z_{0}, z_{1}, \ldots, z_{i+1}$. It follows that $P\left(z_{k}\right)$ is strictly shorter than $P(u)$, a contradiction. Now suppose that $k=i-2$ and $z_{t}$ is a neighbor of $v$. In this case, 
$z_{i-2}$ and $z_{j}$ must be adjacent, i.e., $j=i-3$. If $z_{j-1}$ is also a neighbor of $v$, then $\beta_{G}\left(z_{i-2}\right)=d_{G}(v) \leq f(\Delta)$, and we are done.

Suppose that $z_{j-1}$ is not a neighbor of $v$. If there is at least one vertex $z_{p}$, $0<p<j-1$, that is adjacent to $v$, then there is some 2-vertex $z_{m}, p<m<j$, such that all the vertices at distance at most 2 from $z_{m}$ are included in the path $z_{0}$, $z_{1}, \ldots, z_{i-1}$. Therefore, $P\left(z_{m}\right)$ is strictly shorter than $P(u)$, a contradiction.

Now suppose that no such $z_{p}$ exists. If $d_{G}(v) \geq 7$, or $d_{G}(w) \geq 5$, or $d_{G}(w)=4$ but $w$ is not adjacent to $z_{t}$, then there is some $z_{s}, i+2<s<t$, that is a neighbor of $v$ or $w$. It follows that there is some 2-vertex $z_{m}, i+2 \leq m<s$, such that all the vertices at distance at most 2 from $z_{m}$ are included in the path $z_{i-1}, z_{i}, \ldots$, $z_{t}$. Therefore, $P\left(z_{m}\right)$ is strictly shorter than $P(u)$, a contradiction. The remaining possibilities are such that $d_{G}(v)=6$ and $d_{G}(w)=3$, or $d_{G}(w)=4$ and $w$ is adjacent to $z_{t}$. We see that $\beta_{G}(u)=7 \leq f(\Delta)$ in both cases and $u$ satisfies the theorem.

Subcase 2.2. The vertex $z_{0}$ is precisely $x_{v}$.

If $z_{t}$ is a neighbor of $w$, then $\beta_{G}(u) \leq 5 \leq f(\Delta)$ since $3 \leq d_{G}(w) \leq d_{G}(v) \leq 4$ in this case. Suppose that $z_{t}$ is adjacent to $v$, but not to $w$. It is obvious that $d_{G}(v) \geq 4$. If $d_{G}(w) \geq 4$, then there is some $z_{j}, i+2<j<t$, that is adjacent to $w$. Thus there is some 2 -vertex $z_{k}, i+2 \leq k<j$, such that all the vertices at distance at most 2 from $z_{k}$ are included in the path $z_{i-1}, z_{i}, \ldots, z_{t}$. It follows that $P\left(z_{k}\right)$ is strictly shorter than $P(u)$, a contradiction. So suppose $d_{G}(w)=3$. If $d_{G}(v)=4$, then $\beta_{G}(u)=5 \leq f(\Delta)$, hence $u$ satisfies the theorem. If $d_{G}(v) \geq 5$, then there is some $z_{p}, i+2<p<t$, that is adjacent to $v$. Since $v$ is not adjacent to $x_{w}$, there is some 2 -vertex $z_{q}, i+2 \leq q<p$, such that all the vertices at distance at most 2 from $z_{q}$ are included in the path $z_{i-1}, z_{i}, \ldots, z_{t}$. It follows that $P\left(z_{q}\right)$ is strictly shorter than $P(u)$, a contradiction.

Subcase 2.3. The vertex $z_{0}$ is different from $x_{v}$ and is a neighbor of $v$ such that no vertices among $z_{j}, 0<j<i-2$, are adjacent to $v$.

First assume that $z_{t}$ is not a neighbor of $v$. Thus $d_{G}(v)=4$. If $d_{G}(w)=3$, then $\beta_{G}(u)=5 \leq f(\Delta)$. If $d_{G}(w)=4$, then $\beta_{G}(u)=6 \leq f(\Delta)$ when $\Delta \geq 5$. In both cases, $u$ satisfies the theorem.

Now assume that $\Delta=4$. If the degree of $z_{i-2}$ is 4 , or is 3 but $z_{i-2}$ is not a neighbor of $z_{0}$, then some $z_{j}, 0<j<i-2$, is a neighbor of $z_{i-2}$. Thus there is some 2-vertex $z_{k}, j<k<i-2$, such that all the vertices at distance at most 2 from $z_{k}$ are included in the path $z_{0}, z_{1}, \ldots, z_{i-1}$. It follows that $P\left(z_{k}\right)$ is strictly shorter than $P(u)$, a contradiction.

Suppose that $z_{i-2}$ is of degree 3 and adjacent to $z_{0}$. If $z_{i-3}$ is of degree 2 and adjacent to $z_{0}$, then $\beta_{G}\left(z_{i-3}\right)=4<f(\Delta)$. Thus $z_{i-3}$ satisfies the theorem. If $z_{i-3}$ is of degree 2 , but not adjacent to $z_{0}$, then all the vertices at distance at most 
2 from $z_{i-3}$ are included in the path $z_{0}, z_{1}, \ldots, z_{i-1}$. It follows that $P\left(z_{i-3}\right)$ is strictly shorter than $P(u)$, a contradiction.

Suppose that the degree of $z_{i-3}$ is at least 3 . Then $z_{i-3}$ cannot be a neighbor of $z_{0}$, for otherwise $v$ would be a cut vertex. Then there is some 2 -vertex $z_{k}$, $1<k<i-3$, such that all the vertices at distance at most 2 from $z_{k}$ are included in the path $z_{0}, z_{1}, \ldots, z_{i-2}$. It follows that $P\left(z_{k}\right)$ is strictly shorter than $P(u)$, a contradiction.

Consequently, the only possibility left for $z_{i-2}$ is its degree is 2 . If $z_{i-2}$ is not adjacent to $z_{0}$, then all the vertices at distance at most 2 from $z_{i-2}$ are included in the path $z_{0}, z_{1}, \ldots, z_{i+1}$. It follows that $P\left(z_{i-2}\right)$ is strictly shorter than $P(u)$, a contradiction. If $z_{i-2}$ is adjacent to $z_{0}$, i.e., $i=3$, and $d_{G}\left(z_{0}\right)=3$, then $\beta_{G}\left(z_{1}\right)=5=f(\Delta)$. Thus $z_{1}$ satisfies the theorem. So the last remaining possibility is that $z_{1}$ is a 2 -vertex, $z_{1}$ is adjacent to $z_{0}$, and $z_{0}$ is a 4 -vertex.

Now since $d_{G}(v)=d_{G}(w)=4$, an argument similar to the above for $z_{i-2}$ can be applied to $z_{5}$. We either obtain a desired 2-vertex or the degrees of $z_{5}$ and $z_{6}$ are 2 and 4 , respectively. Note that the vertices $z_{0}, z_{1}, \ldots, z_{6}$ would induce a configuration $M_{3}$. However, that is ruled out by the assumptions of the theorem.

Finally, suppose that $z_{t}$ is a neighbor of $v$. This implies that $d_{G}(v) \geq 5$. If $d_{G}(v) \geq 6$, or $d_{G}(w) \geq 5$, or if $d_{G}(v)=5, d_{G}(w)=4$, and $z_{t}$ is not adjacent to $w$, then there is some $z_{j}, i+2<j<t$, that is adjacent to $v$ or $w$. It follows that there is some 2-vertex $z_{k}, i+2 \leq k<j$, such that all the vertices at distance at most 2 from $z_{k}$ are included in the path $z_{i-1}, z_{i}, \ldots, z_{t}$. Therefore, $P\left(z_{k}\right)$ is strictly shorter than $P(u)$, a contradiction. If $d_{G}(v)=5$, and $d_{G}(w)=3$ or $d_{G}(w)=4$ but $z_{t}$ is adjacent to $w$, then $\beta_{G}(u)=6 \leq f(\Delta)$. Thus $u$ satisfies the theorem.

Let $C_{5}+e$ be the graph obtained from a cycle of length 5 with two nonconsecutive vertices joined. Then its maximum degree is 3 and $\beta_{C_{5}+e}(u)=4$ for any vertex $u$.

For $n \geq 3$, let $O_{n}$ denote the outerplane graph obtained by adding $n$ edges $u_{1} u_{2}, u_{2} u_{3}, \ldots, u_{n} u_{1}$ inside a cycle $u_{1}, v_{1}, u_{2}, v_{2}, \ldots, u_{n}, v_{n}, u_{1}$ of length $2 n$. We have $\Delta\left(O_{3}\right)=4$ and $\beta_{O_{3}}(u)=5$ for any vertex $u$. Let $A$ be the graph obtained from $O_{4}$ by joining the vertices $u_{1}$ and $u_{3}$. Then $\Delta(A)=5$ and $\beta_{A}(u)=6$ for any 2-vertex $u$. Let $B$ be the graph obtained from $O_{6}$ by adding the new triangle $u_{1} u_{3} u_{5}$. Then $\Delta(B)=6$ and $\beta_{B}(u)=7$ for any 2 -vertex $u$. Therefore, the upper bound in Theorem 1 cannot be further reduced when $3 \leq \Delta \leq 6$.

\section{Coloring the Square}

Let $G$ be a connected graph. It is straightforward to verify the following facts.

(1) If $\Delta(G)=1$, then $\chi\left(G^{2}\right)=2$. 
(2) If $\Delta(G)=2$ and $G$ is a path, then $\chi\left(G^{2}\right)=3=\Delta(G)+1$. If $\Delta(G)=2$ and $G$ is a cycle, then $3 \leq \chi\left(G^{2}\right) \leq 5$. Moreover, $\chi\left(G^{2}\right)=3=\Delta(G)+1$ if and only if $|G| \equiv 0(\bmod 3) ; \chi\left(G^{2}\right)=5=\Delta(G)+3$ if and only if $|G|=5$.

Lemma 2. Let $x$ be a cut vertex of the graph $G$. Let $G_{i}$ be the subgraph induced by $V_{i} \cup\{x\}$ for $i=1,2, \ldots, m$, where $V_{i}$ 's are the vertex sets of the components of $G-x$. Then $\chi\left(G^{2}\right)=\max _{1 \leq i \leq m}\left\{d_{G}(x)+1, \chi\left(G_{i}^{2}\right)\right\}$.

Proof. Let $k=\max _{1 \leq i \leq m}\left\{d_{G}(x)+1, \chi\left(G_{i}^{2}\right)\right\}$. Since $G_{i}$ is a subgraph of $G, \chi\left(G^{2}\right) \geq \chi\left(G_{i}^{2}\right)$ for every $i, 1 \leq i \leq m$. Moreover, it is obvious that $\chi\left(G^{2}\right) \geq \Delta(G)+1 \geq d_{G}(x)+1$. It follows that $\chi\left(G^{2}\right) \geq k$. Conversely, let each $G_{i}$ be colored with a square- $\chi\left(G_{i}^{2}\right)$-coloring. Then all the neighbors of $x$ in $G_{i}$ have different colors. By suitably renaming the colors, we can color $x$ with the same color in every $G_{i}$ and all the neighbors of $x$ in $G$ are colored differently. It follows that $k \geq \chi\left(G^{2}\right)$.

Theorem 3. Let $G$ be an outerplane graph with $\Delta(G) \geq 3$. Then $\chi\left(G^{2}\right) \leq$ $\Delta(G)+2$. Moreover, $\chi\left(G^{2}\right)=\Delta(G)+1$ if $\Delta(G) \geq 7$.

Proof. We proceed by induction on the order $|G|$. We may suppose the connectedness of $G$. If $|G| \leq 4$, the theorem holds trivially. Let $\Delta(G) \geq 3$ and $|G| \geq 5$.

Suppose that $G$ is 2-connected. If $\Delta(G) \neq 4$, or $\Delta(G)=4$ but $G$ does not contain the configuration $M_{3}$, then there is a 2-vertex $u$ of $G$ such that $\beta_{G}(u) \leq$ $\Delta(G)+1$ by Theorem 1 . Let $v$ and $w$ be the neighbors of $u$. If $v$ and $w$ are not adjacent, define $H$ to be $G-u+v w$. If $v$ and $w$ are adjacent, define $H$ to be $G-u$. Then $|H|<|G|, \Delta(H)=\Delta(G)$, and $H$ is 2-connected. By the induction hypothesis, $H$ has a square- $(\Delta(G)+2)$-coloring. We can extend this coloring to $G$ since the vertex $u$ has at most $\Delta(G)+1$ forbidden colors.

Now let $\Delta(G)=4$ and $G$ contains the configuration $M_{3}$. Let $y_{1}, y_{2} \in N_{1}\left(x_{1}\right) \backslash$ $\left\{x_{2}, x_{3}\right\}$ and $z_{1}, z_{2} \in N_{1}\left(x_{7}\right) \backslash\left\{x_{5}, x_{6}\right\}$. If $x_{1}$ is adjacent to $x_{7}$, we stipulate that $y_{2}=x_{7}$ and $z_{2}=x_{1}$. Define the graph $H$ to be $G-\left\{x_{2}, x_{3}, \ldots, x_{6}\right\}$ if $x_{1}$ is adjacent to $x_{7}$; to be $G-\left\{x_{2}, x_{3}, \ldots, x_{6}\right\}+x_{1} x_{7}$ otherwise. By the inductive hypothesis, $H$ has a square-6-coloring $\phi$ with the color set $L=\{1,2, \cdots, 6\}$. In order to extend $\phi$ into a square-6-coloring of $G$, we consider the following two cases.

Assume that $x_{1}$ is adjacent to $x_{7}$. Without loss of generality, we may let $\phi\left(y_{1}\right)=$ $1, \phi\left(x_{1}\right)=2, \phi\left(x_{7}\right)=3$, and $\phi\left(z_{1}\right)=a$. We first color $x_{4}$ with $1, x_{5}$ with $b \in L \backslash\{1,2,3, a\}$, and $x_{2}$ and $x_{6}$ with $c \in L \backslash\{1,2,3, a, b\}$. Afterward, we assign $a$ to $x_{3}$ when $a \neq 1$; we color $x_{3}$ with $d \in L \backslash\{1,2,3, b, c\}$ when $a=1$.

Assume that $x_{1}$ is not adjacent to $x_{7}$. Since $x_{1}$ is adjacent to $x_{7}$ in $H, \phi\left(x_{1}\right) \notin$ $\left\{\phi\left(z_{1}\right), \phi\left(z_{2}\right)\right\}$ and $\phi\left(x_{7}\right) \notin\left\{\phi\left(y_{1}\right), \phi\left(y_{2}\right)\right\}$. Suppose that $\phi\left(y_{1}\right)=1, \phi\left(y_{2}\right)=2$, 
$\phi\left(x_{1}\right)=3$, and $\phi\left(x_{7}\right)=4$. First we color $x_{4}$ with $1, x_{6}$ with $3, x_{2}$ with 4, and $x_{3}$ with 5. If 2 or $6 \notin\left\{\phi\left(z_{1}\right), \phi\left(z_{2}\right)\right\}$, we further color $x_{5}$ with 2 or 6 . If $\left\{\phi\left(z_{1}\right), \phi\left(z_{2}\right)\right\}=\{2,6\}$, we recolor $x_{4}$ with 6 and then color $x_{5}$ with 1 .

Next suppose that $G$ has a cut vertex $x$. Let $G_{i}, 1 \leq i \leq m$, be the subgraphs induced by the components of $G-x$ together with $x$. Then each $G_{i}$ satisfies the assumptions of the theorem. If $\Delta\left(G_{i}\right) \geq 3$, then $\chi\left(G_{i}^{2}\right) \leq \Delta\left(G_{i}\right)+2 \leq \Delta(G)+2$ by the induction hypothesis. If $\Delta\left(G_{i}\right) \leq 2$, then $\chi\left(G_{i}^{2}\right) \leq 5 \leq \Delta(G)+2$ as noted at the beginning of this section. Thus $\chi\left(G^{2}\right) \leq \Delta(G)+2$ by Lemma 2 .

The "moreover" part can also be proved by induction since the 2-vertex $u$ could have been chosen so that $\beta_{G}(u) \leq \Delta(G)$ by Theorem 1 .

It is yet to be determined if any outerplanar graph $G$ with $\Delta(G)=5$ or 6 satisfies $\chi\left(G^{2}\right)=\Delta(G)+2$. We would conjecture that none exists. If an outerplanar graph $G$ with $\Delta(G)=3$ contains a 5 -cycle, then $\chi\left(G^{2}\right)=5=\Delta(G)+2$. This example together with the following theorem shows that the upper bound $\Delta(G)+2$ in Theorem 3 is tight for $\Delta(G)=3$ or 4 .

Theorem 4. For any $n \geq 3, \chi\left(O_{n}^{2}\right)=5$ except $\chi\left(O_{3}^{2}\right)=\chi\left(O_{4}^{2}\right)=\chi\left(O_{7}^{2}\right)=6$.

Proof. It is easy to see that $5 \leq \chi\left(O_{n}^{2}\right) \leq 6$ for every $n \geq 3$. Since $O_{3}^{2}$ is $K_{6}$ and $O_{4}^{2}$ contains $K_{6}$ as a subgraph, we have $\chi\left(O_{3}^{2}\right)=\chi\left(O_{4}^{2}\right)=6$. We observe that every color class contains at most three vertices for a square- $k$-coloring of $O_{7}$. If a color class is of size 3, then it contains at least two vertices of degree 2. Since $O_{7}$ has seven vertices of degree 2 , there are at most three color classes of size 3 . This implies $k \geq 6$ and $\chi\left(O_{7}^{2}\right)=6$.

Now assume $n \geq 5$ and $n \neq 7$. We are going to construct a square-5-coloring of $O_{n}$ in every possible case.

If $n \equiv 0(\bmod 5)$, we color the sequence of vertices $u_{1}, v_{1}, u_{2}, v_{2}, \ldots, u_{n}, v_{n}$ with the color sequence $1,2,3,4,5$ repeatedly.

If $n \equiv 1(\bmod 5)$, we first color $u_{1}$ and $u_{4}$ with $1, u_{2}$ and $u_{5}$ with $2, u_{3}$ and $u_{6}$ with $3, v_{1}, v_{3}, v_{5}$ with 4 , and $v_{2}, v_{4}, v_{6}$ with 5 . Then we color the sequence of vertices $u_{7}, v_{7}, u_{8}, v_{8}, \ldots, u_{n}, v_{n}$ with the color sequence $1,4,2,3,5$ repeatedly.

If $n \equiv 2(\bmod 5)$ and $n \geq 12$, we first color $u_{1}, u_{4}, u_{7}, u_{10}$ with $1, u_{2}, u_{5}, u_{8}, u_{11}$ with $2, u_{3}, u_{6}, u_{9}, u_{12}$ with $3, v_{1}, v_{3}, v_{5}, v_{7}, v_{9}, v_{11}$ with 4 , and $v_{2}, v_{4}, v_{6}, v_{8}, v_{10}, v_{12}$ with 5 . Then we color the sequence of vertices $u_{13}, v_{13}, u_{14}, v_{14}, \ldots, u_{n}, v_{n}$ with the color sequence $1,4,2,3,5$ repeatedly.

If $n \equiv 3(\bmod 5)$, we first color $v_{1}, v_{3}, v_{5}, v_{7}$ with $1, u_{1}, u_{4}, v_{6}$ with $2, u_{2}, v_{4}, u_{7}$ with $3, v_{2}, u_{5}, u_{8}$ with 4 , and $u_{3}, u_{6}, v_{8}$ with 5 . Then we color the sequence of vertices $u_{9}, v_{9}, u_{10}, v_{10}, \ldots, u_{n}, v_{n}$ with the color sequence $2,3,1,4,5$ repeatedly.

If $n \equiv 4(\bmod 5)$, we first color $u_{1}, u_{4}, u_{7}$ with $1, u_{2}, v_{4}, v_{6}, v_{8}$ with 2 , $v_{2}, u_{5}, v_{7}, v_{9}$ with $3, v_{1}, v_{3}, v_{5}, u_{8}$ with 4 , and $u_{3}, u_{6}, u_{9}$ with 5 . Then color the 
sequence of vertices $u_{10}, v_{10}, u_{11}, v_{11}, \ldots, u_{n}, v_{n}$ with the color sequence $1,4,2$, 5,3 repeatedly.

\section{REFERENCES}

1. G. Agnarsson and M. M. Halldorsson, Coloring powers of planar graphs, SIAM J. Discrete Math. 16 (2003), 651-662.

2. O. V. Borodin, H. J. Broersma, A. Glebov, and J. van den Heuvel, Stars and bunches in planar graphs. part II: general planar graphs and colourings, CDAM Research Report, London School of Economics and Politics, 2002, a translated and adapted version of a paper that appeared in Diskretn. Anal. Issled. Oper. Ser. 1., 8 (2001), 9-33 (in Russian).

3. H. L. Bodlaender, T. Kloks, R. B. Tan and J. van Leeuwen, $\lambda$-coloring of graphs, STACS 2000 (Lille), Lecture Notes in Comput. Sci. 1770, Springer, Berlin, 2000, 395-406.

4. J. van den Heuvel and S. McGuinness, Coloring the square of a planar graph, $J$. Graph Theory 42 (2003), 110-124.

5. M. Molloy and M. R. Salavatipour, A bound on the chromatic number of the square of a graph, preprint, University of Toronto, 2002.

6. C. Thomassen, Applications of Tutte cycles, preprint, Technical University of Denmark, 2001.

7. Weifan Wang and Ko-Wei Lih, Labeling planar graphs with conditions on girth and distance two, SIAM J. Discrete Math., 17 (2003), 264-275.

8. G. Wegner, Graphs with given diameter and a coloring problem, preprint, University of Dortmund, 1977.

Ko-Wei Lih

Institute of Mathematics,

Academia Sinica,

Nankang, Taipei 115, Taiwan

E-mail: makwlih@sinica.edu.tw

Wei-Fan Wang

Department of Mathematics,

Zhejiang Normal University,

Jinhua, Zhejiang 321004, P. R. China 